\title{
BMJ Open Do pregnant women living in higher well-being populations in the USA experience lower risk of preterm delivery? A cross-sectional study
}

Carley Riley, ${ }^{1,2}$ Brita Roy, ${ }^{3}$ Jeph Herrin, ${ }^{3}$ Erica Spatz, ${ }^{3}$ Mark T Silvestri, ${ }^{\odot 4}$
Anita Arora, ${ }^{\circ}$ Kenneth P Kell, ${ }^{5}$ Elizabeth Y Rula, ${ }^{5}$ Harlan M Krumholz ${ }^{\oplus, 6,7}$

To cite: Riley C, Roy B, Herrin J, et al. Do pregnant women living in higher well-being populations in the USA experience lower risk of preterm delivery? A crosssectional study. BMJ Open 2019;9:e024143. doi:10.1136/ bmjopen-2018-024143

- Prepublication history for this paper is available online. To view these files, please visit the journal online (http://dx.doi. org/10.1136/bmjopen-2018024143).

Received 11 May 2018 Revised 24 January 2019 Accepted 18 February 2019

\section{ABSTRACT}

Objective To determine if preterm birth, defined as gestational age $<37$ weeks, is lower for women living in counties with higher well-being, after accounting for known individual risk factors.

Design Cross-sectional study of all US births in 2011. Participants We obtained birth data from the National Center for Health Statistics which included 3938985 individuals.

Main outcomes measures Primary outcome measure was maternal risk of preterm delivery by county; primary independent variable was county-level well-being as measured by the Gallup-Sharecare Well-Being Index (WBI). Results Women living in counties with higher population well-being had a lower rate of preterm delivery. The rate of preterm birth in counties in the lowest WBI quintile was $13.1 \%$, while the rate of preterm birth in counties in the highest WBI quintile was $10.9 \%$. In the model adjusted for maternal risk factors (age, race, Hispanic ethnicity, smoking status, timing of initiation of prenatal visits, multiparity, maternal insurance payer), the association was slightly attenuated with an absolute difference of $1.9 \%$ (95\% Cl 1.7\% to 2.1\%; $p<0.001$ ).

Conclusions Pregnant women who live in areas with higher population well-being have lower risk of preterm birth, even after accounting for individual risk factors.

\section{INTRODUCTION}

Despite considerable efforts, preterm birth remains a substantial public health problem in the USA. ${ }^{1-3}$ With 1 in 10 babies born at less than 37 weeks gestation, preterm birth is the greatest contributor to infant mortality and a leading cause of long-term neurological disabilities in children, resulting in considerable mortality, morbidity and long-term costs in the USA. ${ }^{4-6}$ Research has demonstrated that while individual factors such as maternal age and smoking status influence a pregnant woman's risk of preterm delivery, ${ }^{3}$ community environment has additional influence. ${ }^{7-9}$

Research has also shown that where a woman lives affects her risk of preterm birth.

\section{Strengths and limitations of this study}

- In this national study, we used two large, unique datasets, including the Gallup-Sharecare Well-being Index and all data on live births in the USA in 2011 from the National Center for Health Statistics.

- With these data, this study was the first to examine whether the average well-being of the population in which a woman lives, defined as a broader, multidimensional, self-reported context of the physical, mental and social health of the geographic community, including the overall life evaluation of its residents, relates to her risk of preterm delivery.

- We summarised rates of preterm birth by quintile of county well-being, and tested for associations between population well-being and gestational age using hierarchical generalised linear models that adjusted for individual maternal risk factors.

- As a cross-sectional study, it cannot assess causation, yet determining whether a relationship exists between population well-being and risk of preterm birth is an essential first step.

- Without an assessment of the well-being of the individual pregnant women, the study cannot determine how population well-being may moderate the effect of women's own well-being or other related individual factors on their risk of preterm delivery.

Decades of study have demonstrated that specific features of the local environment, including neighbourhood poverty, local access to healthy foods and environmental exposures, influence a pregnant woman's risk of preterm delivery. ${ }^{10-14}$ In addition to these features, the way that the community views the overall quality of life in their community is another important, yet often unconsidered, feature of the community. Population well-being is a comprehensive construct that captures these contextual factors and incorporates community members' perceptions of the quality of their life in their community. ${ }^{15} 16$ Well-being at the population or 
community level influences health and well-being at the individual level, with a change in well-being of individuals in a community having an effect on others. ${ }^{17}$ As such, the population well-being of a community within which pregnant women live-defined as a broader, multidimensional, self-reported context of the physical, mental and social health of the geographic community, including the overall life evaluation of its residents-may constitute a more complete and accurate conceptualisation of the environment that influences the women's risk of preterm delivery.

Higher population well-being is not only an outcome worth achieving for its own sake, but also has been associated with other desirable health outcomes, such as greater life expectancy. ${ }^{18}$ If also associated with preterm birth, population well-being may provide a promising novel target for reducing preterm birth rates. Prior studies have examined the relationship of socioeconomic factors with preterm birth, but community well-being is a distinct construct and no study has examined whether the average well-being of the population in which a woman lives relates to her risk of preterm delivery. To address this gap, we used a comprehensive, multidimensional assessment of well-being across the USA, the Gallup-Sharecare Well-Being Index (WBI), previously known as the Gallup-Healthways WBI prior to rebranding following Sharecare's 2016 acquisition of Healthways (Gallup-Sharecare, 2011), and data on all live births in the USA in 2011 (National Center for Health Statistics [NCHS], 2011) to examine whether the rate of preterm birth varies with the overall well-being of the population within which the pregnant woman lives. ${ }^{19}{ }^{20}$ We hypothesised that risk of preterm birth is lower for pregnant women who live in higher well-being populations, even when accounting for known individual maternal risk factors. Such work lays the groundwork for testing whether society-wide interventions to improve well-being might have broad beneficial health effects.

\section{METHODS}

\section{Overview}

We conducted a cross-sectional study in which we linked data on all live births in the USA in 2011 to area-level data on population well-being. Because county was the smallest geographic area available for each mother, we aggregated well-being at the county level as well. Wellbeing was measured at the level of county (or county equivalent) and births were linked to the mother's county of residence. We summarised rates of preterm birth by quintile of county well-being, and tested for associations between population well-being and gestational age using hierarchical generalised linear models that adjusted for maternal risk factors.

\section{Birth data}

Birth data were obtained from NCHS. ${ }^{20}$ The NCHS aggregates and standardises data on births collected from all
50 states and the District of Columbia. We used restricted geocoded special use files from NCHS; these include maternal risk factors, maternal county of residence and gestational age (categorised as $<20$ weeks, 20-27 weeks, 28-31 weeks, 32-33 weeks, 34-36 weeks, 37-38 weeks and $39,40,41$ and 42 or more weeks). This dataset includes geocoding at the county level, using the Federal Information Processing System code. ${ }^{21}$

\section{Study sample}

We included all live births during 2011 where the mother's county of residence was not missing, and for which there were WBI survey responses available from that county. We excluded births with missing gestational age. In 2011, 14 states did not collect information on prenatal visits or payer (AK, AL, AR, AZ, CT, HI, MA, ME, MN, MS, NJ, RI, VA, WV). We did not impute missing data due to non-random missingness and likely confounding with the outcome. These missing variables were instead coded as unknown; in sensitivity analyses, we omitted these states.

\section{Outcome}

Our primary outcome was preterm delivery, defined as gestational age $<37$ weeks. $^{22}$

\section{Population well-being data}

Our primary independent variable was population wellbeing. Data on population well-being were obtained from the Gallup-Sharecare WBI survey for $2011 .{ }^{19}$ To develop the WBI, survey items that aligned with prior research on well-being were compiled by experts in the field. ${ }^{23-25}$ Based on the existing literature, items were selected so that the survey would include both hedonic well-being (ie, people's feelings and thoughts about their lives) and eudemonic well-being (ie, an individual's judgements about the meaning and purpose in one's life). ${ }^{26}$ The survey therefore includes items assessing daily emotional experience and a wide variety of evaluative domains, such as overall life, standard of living and satisfaction with community, work, relationships and personal health. Data from a large, representative national sample was then used to perform factor analysis to determine the final set of questions. Criterion validity of geographically aggregated data was established by examining correlations with health and socioeconomic indicators. ${ }^{27}$ Principal component and confirmatory factor analyses were then used to create an instrument valid for measuring individual wellbeing. The individual well-being measure has acceptable reliability, internal and external validity. ${ }^{28}$

In 2011, the WBI comprised 55 self-reported items organised into six domains: life evaluation, emotional health, physical health, healthy behaviours, basic access and work environment. ${ }^{24}$ The Life Evaluation Index measures life satisfaction and optimism about the future. The Emotional Health Index measures daily emotions and the presence or absence of depression. The Physical Health Index assesses the burden of chronic disease and recent illness. The Healthy Behaviours Index assesses the 
prevalence of smoking, exercising and eating fruit and vegetables. The Basic Access Index includes perception of safety and access to housing and healthcare. The Work Environment Index assesses job satisfaction, trust and respect in the workplace and, unlike the other domains, it is collected only from the subset of respondents who report being employed. Each domain is represented by an index, measured on a scale of 0 to 100. A composite score, the WBI, is calculated as the unweighted mean of the six domain scores, and is reported on a scale of $0-100$. Gallup surveyed a unique sample of nearly 1000 individuals 18 years and older every day for approximately 350 days during 2011. A structured sampling design was used with respondents surveyed from all 50 states and the District of Columbia. The survey was administered in both English and Spanish, using both land lines and cell phones. We aggregated individual WBI responses into county scores based on the maternal county of residence. For our primary analyses, we used quintiles of county WBI scores as our independent variable; in secondary analyses, we used county-level quintiles for each of the individual domain scores.

\section{Other independent variables}

From the NCHS birth dataset we included the following known maternal risk factors for preterm delivery: age (categorised as $\leq 19,20-24,25-29,30-34,35-39,40+$ ), race (white, black, Asian, American Indian/Alaska native), Hispanic ethnicity, smoking status, start of prenatal visits (first trimester, second trimester, third trimester, none, not known) and multiparity (single birth vs multiple). We also included infant sex, and, as a marker of socioeconomic status (SES), we included the maternal insurance payer (Medicaid, private, self, other, unknown).

\section{Statistical analysis}

We summarised the outcome, WBI score and all maternal risk factors by quintile of population WBI, reporting frequency and per cent of births in each category. To assess the association between population well-being and preterm birth, we estimated two individual level mixed effects linear models. Both models had the same dichotomous outcome (preterm birth) and both included a random intercept for county. Though logistic regression models are conventionally used for dichotomous outcomes, linear probability models such as these are appropriate when the outcome rate is not close to 0 or 1 , and the predicted values from the model are also between 0 and 1 . One advantage of using a linear model is that the intercept and coefficients have direct interpretations as a reference rate and risk differences, respectively. The first model was unadjusted, including only county quintile of population well-being. The second adjusted for maternal age, race, ethnicity, smoking status, trimester during which prenatal care was initiated, single or multiple birth, and insurance payer. For both models we calculated the Wald $p$ value for the overall effect of WBI and a separate test for trend in effects across WBI quintiles. In secondary analyses, we replicated the main analyses using each of the six domain scores of the WBI.

We also estimated a reference model which included no independent variables, and used the county level variance from this model to calculate the variance explained at the county level for each of the models described above, using $\mathbf{R}^{2}=\left(\tau^{2}-\tau^{*^{2}}\right) / \tau^{2}$, where $\tau^{*^{2}}$ is the county level variance for the model with independent variables. ${ }^{29}$

All analyses were performed using Stata V.14.2 (2016 StataCorp).

\section{Patient and public involvement}

No patients or the public were involved in the planning and design of this study.

\section{RESULTS}

We used data from 3938985 births across 2989 counties, representing $99.6 \%$ of all US births in 2011 . The mean (SD) county-level preterm birth rate was $11.7 \%(2.2 \%)$ preterm births. Table 1 shows the numbers and percentages of children born before and after 37 weeks gestation, maternal characteristics and infant sex by quintile of average well-being of the maternal county of residence.

The observed rate of preterm birth decreased across WBI quintiles from $13.1 \%$ in the lowest quintile to $10.9 \%$ in the highest quintile (table 1). In the unadjusted model, pregnant women living in counties with higher population well-being had a significantly lower risk of preterm birth, $-0.2 .2 \%$ (95\% CI: $-2.6 \%$ to $-1.8 \%$; $\mathrm{p}<0.001$ ) (table 2 ). After adjusting for maternal risk factors for preterm birth, the trend remained consistent across the quintiles; the absolute difference between the highest and lowest quintiles was attenuated to $-1.9 \%$ (95\% CI: $-2.2 \%$ to $-1.6 \%$; $\mathrm{p}<0.001)$. In sensitivity analyses, results were similar.

In secondary analyses, independent associations between quintiles of each well-being domain and preterm birth are reported in table 3 . Similar to the composite WBI score, all domain scores were significantly associated with maternal risk of preterm birth in the unadjusted model and the model adjusting for individual maternal risk factors. Different domains, however, explained different amounts of variance of well-being, with the basic access index explaining $14.6 \%$ of the county variance. After adjusting for maternal risk factors, women in counties with the highest basic access score experienced an absolute difference in preterm birth rates of $-2.4 \%$ (95\% CI: $-2.2 \%$ to $-2.6 \% ; \mathrm{p}<0.001)$ when compared with women in counties with the lowest basic access score. Similarly, the average physical health score of the county within which a pregnant woman resided was associated with lower rates of preterm birth, in both unadjusted and adjusted models (absolute difference in preterm birth rate: $-1.9 \%, 95 \%$ CI: $-1.6 \%$ to $-2.1 \%$; $p<0.001)$. In the models adjusted for maternal risk factors, healthy behaviours $(-1.5 \%, 95 \%$ CI: $-1.2 \%$ to $-1.8 \%$; $\mathrm{p}<0.001)$, emotional health $(-1.0 \%, 95 \%$ CI: $-0.7 \%$ to $-1.3 \%$; $\mathrm{p}<0.001)$ and life evaluation $(-1.1 \%, 95 \%$ CI: $-0.8 \%$ to $-1.4 \%$; $\mathrm{p}<0.001)$ of 


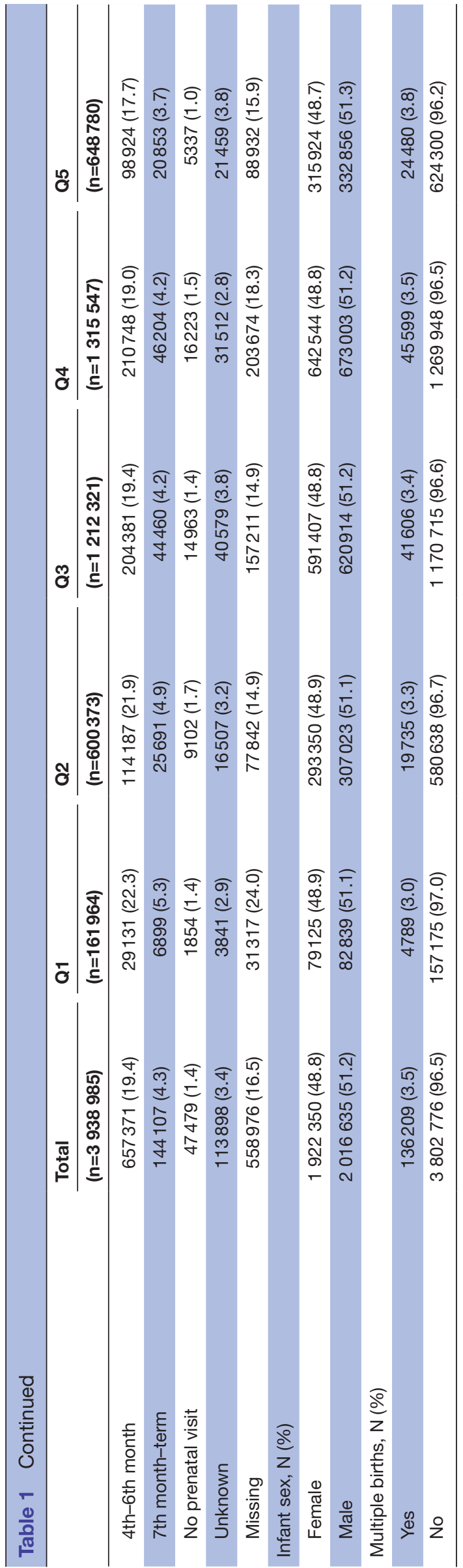

the county population negatively correlated with risk of preterm birth.

\section{DISCUSSION}

In this study of nearly all births in the USA in 2011, we found that pregnant women who lived in counties with higher average well-being had significantly lower risk of preterm birth. After accounting for known maternal risk factors, including age, race, ethnicity, smoking status, timing of initiation of prenatal care, multiparity and payer type, the absolute difference in maternal risk for preterm delivery between the highest well-being counties and the lowest well-being counties was $1.9 \%$. If this relationship is causal, and if the lowest well-being counties experienced this reduced rate, they would have had 3077 fewer preterm births in 2011 alone, yielding an estimated financial savings of nearly $\$ 160$ million, ${ }^{30}$ in addition to fewer long-term consequences of preterm birth such as infant mortality and long-term disabilities. Additional reductions in preterm birth risk in counties from the middle well-being quintiles which contributed greater total numbers of births in 2011, would augment these benefits.

Decades of research have delineated a variety of maternal-level risk factors for preterm delivery, including maternal age, smoking status, history of preterm delivery and SES, ${ }^{2} 31$ while recent studies of environmental factors suggest that features of where a pregnant woman lives, including neighbourhood poverty, local access to healthy foods and environmental exposures, additionally influence her risk of preterm delivery. ${ }^{10} 111314$ Our study extends the existing literature by leveraging a large national data sources to assess how a comprehensive, multidimensional measure of population well-being, including the self-reported overall quality of life, and its various domains relate to individual preterm birth risk across the USA. In completing this study, we found that pregnant women were at lower risk of preterm delivery when living in higher well-being populations than when living in lower well-being populations, even when we accounted for maternal risk factors that are highly correlated with SES, such as insurance payer, age at time of delivery, smoking status, and timing of first prenatal visit. $^{32} 33$ This finding adds to the growing literature describing the complex interactions between individuals and their local environment, including natural, built and social environments, and their combined effects on health outcomes.

The relationship we identify could plausibly be causal through several mechanisms, including reasons related to the social environment. Pregnant women who live in higher well-being populations may experience less toxic stress, greater access to social resources, higher levels of trust and tolerance, and/or a greater perception of safety. ${ }^{16}$ Prior research has shown that exposure to toxic stress increases the risk of preterm delivery, ${ }^{34-37}$ while stronger social support, less social isolation and greater 
Table 2 Maternal risk of preterm delivery: overall and by county of residence aggregated by quintile of composite GallupSharecare Well-Being Index (WBI) score, unadjusted and adjusted for maternal risk factors

\begin{tabular}{|c|c|c|c|c|c|c|}
\hline Variable & Coefficient $(95 \% \mathrm{Cl})$ & $P$ value & Wald $p$ & Coefficient (SE) & $P$ value & Wald $p$ \\
\hline \multirow[t]{2}{*}{ Intercept } & 0.131 & $<0.001$ & & $0.120(0.118,0.123)$ & $<0.001$ & \\
\hline & (0.128 to 0.134$)$ & & & & & \\
\hline Q1 & ref & & & ref & & \\
\hline Q2 & - & 0.001 & & $-0.006(-0.009,-0.003)$ & $<0.001$ & \\
\hline \multirow[t]{2}{*}{ Q5 } & $-0.015(-0.019$ to 0.012$)$ & $<0.001$ & & $-0.019(-0.022,-0.016)$ & $<0.001$ & \\
\hline & $-0.022(-0.026$ to 0.018$)$ & & & & & \\
\hline
\end{tabular}

\section{Maternal age}

$\leq 19$

20-24

25-29

$30-34$

35-39

$\geq 40$

Maternal race
White
Black
American Indian/Native
American
Asian

Mother is Hispanic

No

Yes

Maternal smoking

No

Yes

Unknown

Payer

Medicaid

Private

Self

Other

Unknown

Timing of first prenatal visit

1st-3rd month

4th-6th month

7th month-term

No prenatal visit

Unknown ref

$\begin{array}{lr}-0.019(-0.020,-0.017) & <0.001 \\ -0.021(-0.022,-0.020) & <0.001 \\ -0.016(-0.017,-0.014) & <0.001 \\ 0.002(0.000,0.003) & 0.018 \\ 0.023(0.021,0.025) & <0.001\end{array}$

ref

$0.051(0.050,0.052) \quad<0.001$

$0.024(0.021,0.027)<0.001$

$0.011(0.009,0.012) \quad<0.001$

ref

$0.015(0.014,0.015) \quad<0.001$

ref

$0.030(0.029,0.031) \quad<0.001$

$0.004(0.002,0.007)$

ref

$\begin{array}{ll}-0.019(0.000) & <0.001 \\ -0.012(0.001) & <0.001 \\ -0.013(0.001) & <0.001 \\ -0.005(0.001) & <0.001\end{array}$

ref

$\begin{array}{ll}-0.019(-0.020,-0.018) & <0.001 \\ -0.012(-0.014,-0.011) & <0.001 \\ -0.013(-0.014,-0.011) & <0.001 \\ -0.005(-0.008,-0.002) & \\ -0.004(-0.006,-0.003) & \end{array}$

Infant sex

Female

ref

Male

$0.011(0.010,0.011)$

$<0.001$ 


\begin{tabular}{|c|c|c|c|c|c|c|}
\hline Variable & Coefficient $(95 \% \mathrm{Cl})$ & $P$ value & Wald $p$ & Coefficient (SE) & $P$ value & Wald $p$ \\
\hline \multicolumn{7}{|c|}{ Multiple births } \\
\hline Yes & & & & $0.487(0.485,0.489)$ & $<0.001$ & \\
\hline $\mathrm{R}^{2}$ & 0.078 & & & 0.656 & & \\
\hline
\end{tabular}

GHWBI, Gallup-Sharecare Well-Being Index.

social connectedness are associated with lower risk of preterm delivery, perhaps by reducing the allostatic load or chronic stress experienced by pregnant women. ${ }^{38-43}$ Additionally, while experiences of perceived discrimination are associated with increased risk of preterm delivery, experiences of trust and tolerance as well as a greater perception of safety may foster healthier pregnancies and term deliveries. ${ }^{44-46}$

According to our results, pregnant women with the same individual maternal risk profile, including factors associated with SES, experience lower risk of preterm delivery when living in higher well-being populations than when living in lower well-being populations. This finding is consistent with emerging epigenetics ${ }^{13} 4748$ and maternal weathering ${ }^{24}{ }^{29}$ literature. The maternal weathering model suggests that certain populations of women have an increased risk of preterm delivery due to 'accelerated ageing' that they experience as a result of greater exposure to hardship. This model suggests that living in better neighbourhoods might attenuate the increased risk associated with these weathering effects. ${ }^{49}$ It is possible that the observed risk contributed by weathering and the risk mitigated by living in higher well-being populations are actually related to underlying exposure to toxic stress and buffering from factors such as trust, tolerance, social support and perceived safety.

Our study also builds on prior literature that found links between living in areas of greater poverty and increased risk of preterm birth. In the domain analyses, the basic access domain demonstrated the strongest relationship with maternal risk of preterm delivery. Even after adjusting for individual maternal risk factors, including insurance provider and smoking, the basic access domain explained nearly $15 \%$ of county-level variance in preterm birth rates. The basic access index domain includes items that assess perceived access to healthcare, clean water, fresh produce and safe public space as well as ability to afford basic needs such as food and shelter. This finding affirms prior literature reporting that access to basic needs is strongly correlated with health outcomes. Importantly, however, all domains contributed independently to the inverse association between population well-being and maternal risk of preterm delivery, though to varying degrees. Average county-level physical health, healthy behaviours and emotional health scores were associated with a $1 \%-2 \%$ lower maternal risk of preterm delivery.
The results of our study have several potential implications. Our findings suggest the possibility that effective population-level and community-level investments in well-being may not only improve overall health and quality of life for populations, but also contribute to reduced rates of preterm birth for pregnant women living in those populations, an idea worth pursuing. Our domain analyses suggest that improving aggregate basic access, in particular, could plausibly result in not only greater well-being but also fewer preterm births. Targeting other domains, such as physical health, healthy behaviours and emotional health, may yield additional improvements. Importantly, the effectiveness of various interventions will most likely depend on the contexts within which they are implemented. Currently, efforts are under way across the globe to track and improve population well-being through programmatic and policy-based interventions. ${ }^{465-52}$ While some interventions involve multisector, community-based programmes, many of which are government supported, other interventions involve changes in economic and social policies, such as those aimed at affordable housing, employment and access to public spaces for physical fitness or social connection. ${ }^{165354}$ Given the relationship between population well-being and preterm birth risk, examining the association of such programmes and policies with preterm birth could be informative and allow for spread of interventions that effectively increase well-being and reduce preterm birth.

Our study has limitations. First, as a cross-sectional study, it cannot assess causation. However, determining whether a relationship exists between population well-being and risk of preterm birth is an essential first step. Second, this study specifically examines how the average well-being of the adult population within which a pregnant woman lives correlates with her risk of preterm delivery. Because we do not have an assessment of the well-being of the individual pregnant women, we cannot determine how population well-being may moderate the effect of women's own wellbeing or other related individual factors on their risk of preterm delivery. Additionally, we did not have data on maternal income, wealth or education level, so we could not directly adjust for these socioeconomic variables. Nevertheless, we used available maternal-level variables that are known to be associated with SES as proxies in order to control for the effect of SES on preterm birth 
Table 3 Maternal risk of preterm delivery by county of residence aggregated by quintile of individual Gallup-Sharecare Well-

Being Index domain scores, unadjusted and adjusted for maternal risk factors

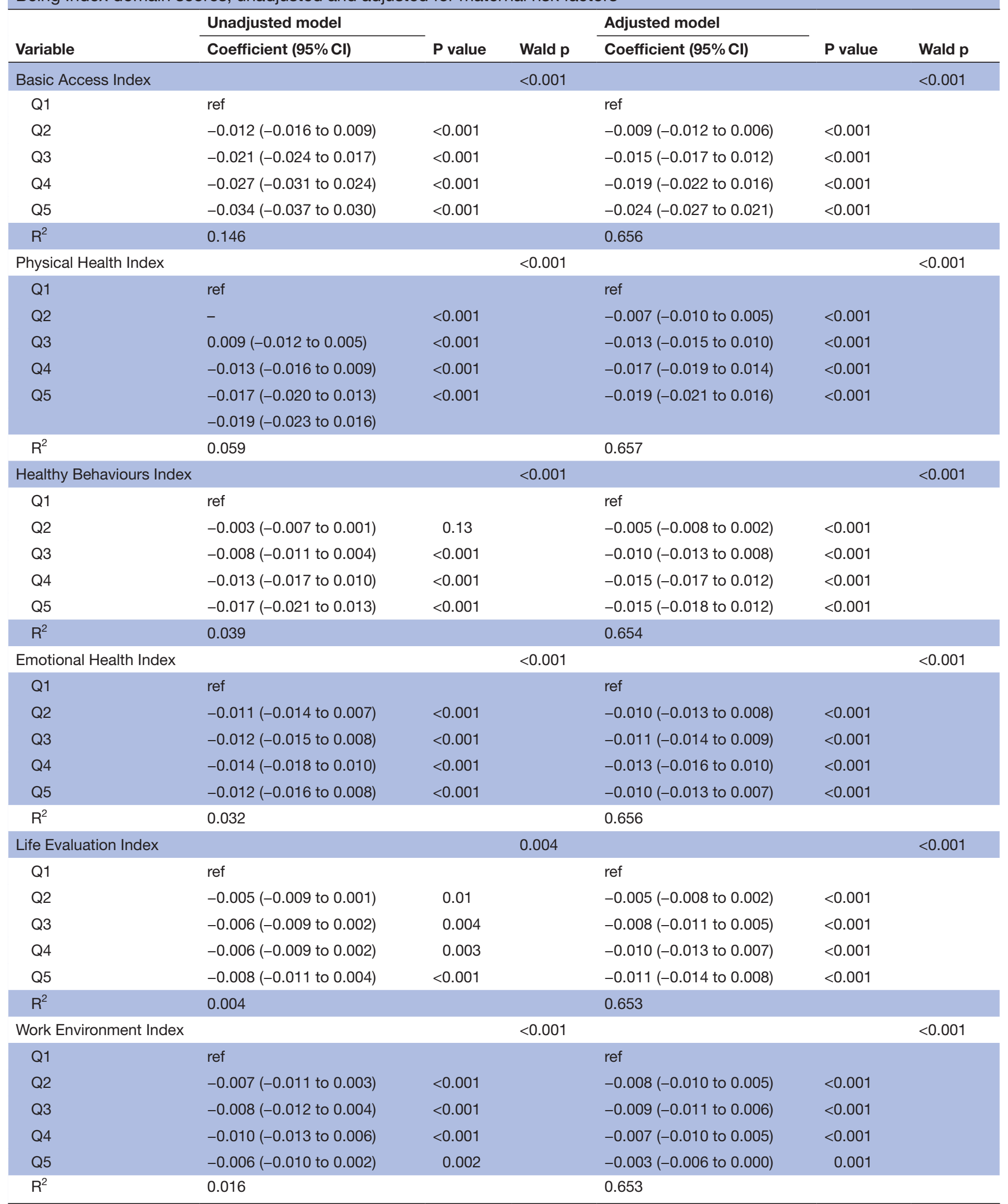


and isolate the effect of community well-being. Finally, we did not have well-being data available at geographically smaller units (eg, neighbourhood or city) which may be more relevant than county well-being in describing the community context for an individual pregnant woman. While counties are distinct from the smaller, often more homogeneous, geographic units of neighbourhoods and census tracts, policies and programmes are often enacted at the county level. Thus, results may drive action at the county-level, while also informing local communities in developing targeted programmes to enhance well-being.

Pregnant women who live in populations with higher well-being have lower risk of preterm delivery, even after accounting for known individual maternal risk factors. The well-being of a population is an important end itself, but if causal pathways exist between population well-being and other valued outcomes, investments in population well-being may yield other benefits, potentially including fewer preterm births. Understanding the full effects of population well-being can inform the emerging dialogue about its value as a health investment.

\section{Author affiliations}

${ }^{1}$ Division of Critical Care, Cincinnati Children's Hospital Medical Center, Cincinnati, Ohio, USA

${ }^{2}$ Department of Pediatrics, University of Cincinnati College of Medicine, Cincinnati, Ohio, USA

${ }^{3}$ Department of Internal Medicine, Yale School of Medicine, New Haven, Connecticut, USA

${ }^{4}$ Department of Obstetrics, Gynecology, and Reproductive Sciences, Yale School of Medicine, New Haven, Connecticut, USA

${ }^{5}$ Tivity Health, Franklin, Tennessee, USA

${ }^{6}$ Center for Outcomes Research and Evaluation, Yale-New Haven Hospital, New Haven, Connecticut, USA

${ }^{7}$ Department of Health Policy and Management, Yale School of Public Health, New Haven, Connecticut, USA

Acknowledgements We would like to thank Brent Hamar, Ashlin Jones, Larissa Loufman, and Allison Parsons for their valuable contributions to the final revision of this manuscript.

Contributors $\mathrm{CR}$ and $\mathrm{BR}$ participated in the initial conception of this study. $\mathrm{JH}$ performed all analyses. All authors (CR, BR, JH, ES, MS, AA, KK, ER, and HK) contributed to the study design, interpretation of data, drafting and revising the article, and its final approval. All authors are guarantors.

Funding This study was supported in part by the Robert Wood Johnson Foundation Clinical Scholars Program.

Competing interests All authors have completed the ICMJE uniform disclosure form at www.icmje.org/coi_disclosure.pdf and declare the following competing interests: partial support from the Agency for Healthcare Research and Quality (BR and ES) and the Robert Wood Johnson Foundation (CR, BR, and AA) and the Veterans Administration (BR) for the submitted work; $C R$ and $B R$ receive funding from the Institute for Healthcare Improvement to support their effort in developing and implementing the measurement framework for the 100 Million Healthier Lives initiative; AA is partially supported by the Yale Center for Clinical Investigation through Clinical and Translational Science Award; ER and KK are current or former employees and shareholders of the Healthways corporation (acquired by Sharecare), the company that developed the measure of well-being used in this article; ES, JH, and HK also report receiving support from the Centers for Medicare and Medicaid Services; Dr. Krumholz is a recipient of research agreements from Medtronic and Johnson \& Johnson (Janssen), through Yale, to develop methods of clinical trial data sharing; was the recipient of a grant from Medtronic and the Food and Drug Administration, through Yale, to develop methods for postmarket surveillance of medical devices; chairs a cardiac scientific advisory board for UnitedHealth; is a participant/participant representative of the IBM Watson Health
Life Sciences Board; is a member of the Advisory Board for Element Science and for FaceBook, and the Physician Advisory Board for Aetna; and is the founder of Hugo, a personal health information platform.

Patient consent for publication Not required.

Ethics approval This study was approved by the Yale University Institutional Review Board.

Provenance and peer review Not commissioned; externally peer reviewed.

Data sharing statement If the paper is accepted for publication, we will post a de-identified data set with county resident well-being data from Gallup-Sharecare on ICSPR Open, a publicly available site. Birth data are available with permission from the National Center for Health Statistics (http://www.cdc.gov/nchs/).

Open access This is an open access article distributed in accordance with the Creative Commons Attribution Non Commercial (CC BY-NC 4.0) license, which permits others to distribute, remix, adapt, build upon this work non-commercially, and license their derivative works on different terms, provided the original work is properly cited, appropriate credit is given, any changes made indicated, and the use is non-commercial. See: http://creativecommons.org/licenses/by-nc/4.0/.

\section{REFERENCES}

1. March of Dimes Premature Birth Report Card. http:// wwwmarchofdimesorg/mission/prematurity-reportcardaspx (Accessed 24 Jan 2017).

2. Muglia LJ, Katz M. The enigma of spontaneous preterm birth. N Engl $J$ Med 2010;362:529-35.

3. Goldenberg RL, Culhane JF, lams JD, et al. Epidemiology and causes of preterm birth. The Lancet 2008;371:75-84.

4. Centers for Disease Control and Prevention. Preterm Birth. http://wwwcdcgov/reproductivehealth/maternalinfanthealth/ pretermbirthhtm.

5. Liu L, Oza S, Hogan D, et al. Global, regional, and national causes of child mortality in 2000-13, with projections to inform post-2015 priorities: an updated systematic analysis. The Lancet 2015;385:430-40.

6. Petrou S, Mehta Z, Hockley C, et al. The impact of preterm birth on hospital inpatient admissions and costs during the first 5 years of life. Pediatrics 2003;112:1290-7.

7. Howard DL, Marshall SS, Kaufman JS, et al. Variations in low birth weight and preterm delivery among blacks in relation to ancestry and nativity: New York City, 1998-2002. Pediatrics 2006;118:e1399-405.

8. David R, Collins J. Disparities in infant mortality: what's genetics got to do with it? Am J Public Health 2007;97:1191-7.

9. Staneva A, Bogossian F, Pritchard M, et al. The effects of maternal depression, anxiety, and perceived stress during pregnancy on preterm birth: a systematic review. Women Birth 2015;28:179-93.

10. Ncube CN, Enquobahrie DA, Albert SM, et al. Association of neighborhood context with offspring risk of preterm birth and low birthweight: A systematic review and meta-analysis of populationbased studies. Soc Sci Med 2016;153:156-64.

11. Brumberg HL, Shah SI. Born early and born poor: an eco-biodevelopmental model for poverty and preterm birth. $J$ Neonatal Perinatal Med 2015;8:179-87.

12. Ma X, Liu J, Hardin JW, et al. Neighborhood food access and birth outcomes in South Carolina. Matern Child Health J 2015;20:187-95.

13. Burris $H H$, Baccarelli $A A$, Wright $R O$, et al. Epigenetics: linking social and environmental exposures to preterm birth. Pediatr Res 2016;79.

14. Love C, David RJ, Rankin KM, et al. Exploring weathering: effects of lifelong economic environment and maternal age on low birth weight, small for gestational age, and preterm birth in African-American and white women. Am J Epidemiol 2010;172:kwq109.

15. World Health Organization. The Ottawa charter for health promotion: first international conference on health promotion, Ottawa, 21 November 1986. Geneva: WHO, 1986.

16. Prilleltensky I. Promoting well-being: Time for a paradigm shift in health and human services. Scand J Public Health 2005:33:53-60.

17. ECW N, Fisher AT. Protestant spirituality and well-being of people in Hong Kong: the mediating role of sense of community. Applied research in quality of life 2016;11:1253-67.

18. Arora A, Spatz E, Herrin J, et al. Population well-being measures help explain geographic disparities in life expectancy at the county level. Health Aff 2016;35:2075-82.

19. Gallup-Healthways. Gallup-Healthways Well-being Index: Methodology Report for Indexes. http://wwwgallupcom/poll/195539/ gallup-healthwaysindex-methodology-report-indexesaspx. 
20. Centers for Disease Control and Prevention. National Center for Health Statistics web site.

21. National Institute of Standards and Technology. Federal Information Processing Standards publications.

22. Tucker J, McGuire W. Epidemiology of preterm birth. BMJ 2004;329:675-8.

23. Kahneman D, Riis J. Living, and thinking about it: two perspectives on life. The science of well-being 2005.

24. Diener E. Guidelines for national indicators of subjective well-being and ill-being. Appl Res Qual Life 2007;1:151-7.

25. Diener E, Seligman ME. Beyond money: toward an economy of wellbeing. Psychol Sci Public Interest 2004;5:1-31.

26. Steptoe A, Deaton A, Stone AA. Subjective wellbeing, health, and ageing. The Lancet 2015;385:640-8.

27. G-HW-b I. Methodology report for indexes. Washington: Gallup Inc, 2009.

28. Evers KE, Prochaska JO, Castle PH, et al. Development of an individual well-being scores assessment. Psychol Well Being 2012;2:2.

29. Kreft IG, Kreft I, de Leeuw J. Introducing multilevel modeling: Sage, 1998.

30. Institute of Medicine Committee on Understanding Premature Birth and Assuring Healthy Outcomes. Preterm birth: causes, consequences, and prevention. 2006.

31. Shah NR, Bracken MB. A systematic review and meta-analysis of prospective studies on the association between maternal cigarette smoking and preterm delivery. Am J Obstet Gynecol 2000;182:465-72.

32. Schempf AH, Branum AM, Lukacs SL, et al. Maternal age and parity-associated risks of preterm birth: differences by race/ethnicity. Paediatr Perinat Epidemiol 2007;21:34-43.

33. Cokkinides V, Bandi P, McMahon C, et al. Tobacco control in the United States--recent progress and opportunities. CA Cancer J Clin 2009;59:352-65.

34. Rubin LP. Maternal and pediatric health and disease: integrating biopsychosocial models and epigenetics. Pediatr Res 2016;79.

35. Premji S. Perinatal distress in women in low- and middle-income countries: allostatic load as a framework to examine the effect of perinatal distress on preterm birth and infant health. Matern Child Health J 2014:18:2393-407.

36. Nkansah-Amankra S, Luchok KJ, Hussey JR, et al. Effects of maternal stress on low birth weight and preterm birth outcomes across neighborhoods of South Carolina, 2000-2003. Matern Child Health J 2010;14:215-26.

37. Dole N, Savitz DA, Hertz-Picciotto I, et al. Maternal stress and preterm birth. Am J Epidemiol 2003;157:14-24.

38. Mendez DD, Hogan VK, Culhane JF. Institutional racism, neighborhood factors, stress, and preterm birth. Ethn Health 2014;19:479-99.
39. Peacock JL, Bland JM, Anderson HR. Preterm delivery: effects of socioeconomic factors, psychological stress, smoking, alcohol, and caffeine. BMJ 1995;311:531-5.

40. Nkansah-Amankra S, Dhawain A, Hussey JR, et al. Maternal social support and neighborhood income inequality as predictors of low birth weight and preterm birth outcome disparities: analysis of South Carolina Pregnancy Risk Assessment and Monitoring System survey, 2000-2003. Matern Child Health J 2010;14:774-85.

41. Seeman TE, Singer BH, Ryff CD, et al. Social relationships, gender, and allostatic load across two age cohorts. Psychosom Med 2002;64:395-406.

42. Brooks KP, Gruenewald T, Karlamangla A, et al. Social relationships and allostatic load in the MIDUS study. Health Psychol 2014;33:1373-81.

43. Collins NL, Dunkel-Schetter C, Lobel M, et al. Social support in pregnancy: psychosocial correlates of birth outcomes and postpartum depression. J Pers Soc Psychol 1993;65:1243-58.

44. Morton J, Withers M, Konrad SC, et al. Bridging the gaps: an early integrated support collaborative for at risk mothers in rural Maine. Work 2015;50:413-23.

45. Morton J, Konrad SC. Introducing a caring/relational framework for building relationships with addicted mothers. J Obstet Gynecol Neonatal Nurs 2009;38:206-13.

46. Konrad S, Morton S, Berzoff J. If I feel judged by you, I will not trust you: relational practice with addicted mothers. falling through the cracks: psychodynamic practice with vulnerable and oppressed populations. 2012.

47. Knight AK, Smith AK. Epigenetic biomarkers of preterm birth and its risk factors. Genes 2016;7:15.

48. Parets SE, Bedient CE, Menon R, et al. Preterm birth and its longterm effects: methylation to mechanisms. Biology 2014;3:498-513.

49. Holzman C, Eyster J, Kleyn M, et al. Maternal weathering and risk of preterm delivery. Am J Public Health 2009;99:1864-71.

50. OECD. How's Life? 2015: Measuring Well-being: OECD Publishing, 2015.

51. Kobau R, Bann C, Lewis M, et al. Mental, social, and physical well-being in New Hampshire, Oregon, and Washington, 2010 Behavioral Risk Factor Surveillance System: implications for public health research and practice related to Healthy People 2020 foundation health measures on well-being. Popul Health Metr 2013;11:19.

52. Derges J, Clow A, Lynch R, et al. 'Well London' and the benefits of participation: results of a qualitative study nested in a cluster randomised trial. BMJ Open 2014;4:e003596.

53. Fowler JH, Christakis NA. Dynamic spread of happiness in a large social network: longitudinal analysis over 20 years in the Framingham Heart Study. BMJ 2008;337:a2338.

54. Diez Roux AV, Mair C. Neighborhoods and health. Ann N Y Acad Sci 2010;1186:125-45. 